TRANSACTIONS OF THE

AMERICAN MATHEMATICAL SOCIETY

Volume 348, Number 1, January 1996

\title{
SUBGROUPS OF GL $\left(n^{2}, \mathbf{C}\right)$ CONTAINING PSU $(n)$
}

\author{
V. P. PLATONOV AND D. Ž. ĐOKOVIĆ
}

\begin{abstract}
Let $\operatorname{PSU}(n)$ be the image of the unitary group $\mathrm{U}(n)$ under the representation $x \rightarrow a x a^{-1}$ on the space $M_{n}(\mathbf{C})$ of $n$ by $n$ complex matrices. We classify all connected Lie subgroups of $\operatorname{GL}\left(n^{2}, \mathbf{C}\right)$ containing $\operatorname{PSU}(n)$. We use this result to obtain a description of all abstract overgroups of $\operatorname{PSU}(n)$ in $\mathrm{GL}\left(n^{2}, \mathbf{C}\right)$.

We apply this classification to solve the problem of describing all invertible linear transformations of $M_{n}(\mathbf{C})$ which preserve the set of normal matrices. Our results can be applied to solve many other problems of similar nature.
\end{abstract}

\section{INTRODUCTION}

The problem of classification of subgroups of the general linear group $\mathrm{GL}(m, \mathbf{C})$ has a long history but, even for Lie subgroups, it is too difficult. For semisimple subgroups, this problem is essentially equivalent to the description of all finite-dimensional representations of such groups. If we are interested only in subgroups having some specific property, then even for semisimple subgroups the problem may be very difficult.

About 20 years ago it was realized, in connection with some problems of invariant theory and linear algebra, that it is important to obtain the classification of subgroups of $\mathrm{GL}(m, \mathbf{C})$ containing a given natural classical subgroup. A well-known problem in this direction is the problem about the description of all algebraic subgroups of $\mathrm{GL}\left(n^{2}, K\right), K$ an algebraically closed field of characteristic 0 , containing the image of $\mathrm{SL}(n, K)$ under the adjoint representation. This problem was solved in our paper [5]. In the present paper we shall solve the problem of describing all abstract subgroups of $\mathrm{GL}\left(n^{2}, \mathbf{C}\right)$ containing the image of $\mathrm{SU}(n)$ under the adjoint representation.

In more detail, let $M_{n}$ be the algebra of $n$ by $n$ complex matrices. The group of all automorphisms of the vector space $M_{n}$ will be denoted by $\operatorname{GL}\left(n^{2}, \mathbf{C}\right)$. We shall consider $M_{n}$ as a left module over the algebra $M_{n} \otimes_{\mathbf{C}} M_{n}^{\text {opp }}$ such that $(a \otimes b)(x)=$ $a x b$. In this way we can identify this algebra with the algebra of all complex linear operators on $M_{n}$.

The group $\operatorname{GL}(n, \mathbf{C})$ acts on $M_{n}$ by similarity transformations $x \rightarrow a x a^{-1}, x \in$ $M_{n}, a \in \mathrm{GL}(n, \mathbf{C})$. The image of $\mathrm{GL}(n, \mathbf{C})$ in $\mathrm{GL}\left(n^{2}, \mathbf{C}\right)$, under this representation, will be denoted by $G$. The image of the unitary group $\mathrm{U}(n) \subset \mathrm{GL}(n, \mathbf{C})$ in $G$ will

Received by the editors August 6, 1994.

1991 Mathematics Subject Classification. Primary 20G20, 15A30.

The first author was supported in part by NSERC Grant A-6197 and the Alexander von Humboldt Foundation.

The second author was supported in part by NSERC Grant A-5285.

(C)1996 American Mathematical Society 
be denoted by $G_{0}$. It is clear that $G \cong \operatorname{PSL}(n, \mathbf{C}), G_{0} \cong \operatorname{PSU}(n)$, and that $G_{0}$ is a compact real form of $G$.

Our main objective is to obtain a description of all abstract subgroups of $\mathrm{GL}\left(n^{2}, \mathbf{C}\right)$ containing $G_{0}$. While this problem is quite natural, it is worthwhile to point out some important applications of this classification.

In our papers [1], [5] we have described all connected algebraic subgroups of $\operatorname{SL}\left(n^{2}, K\right)$ containing $\operatorname{PSL}(n, K)$ for an arbitrary algebraically closed field $K$ of characteristic 0 , and we have applied these results to solve many linear preserver problems (concerning these problems see the survey volume [6]). We shall use here this classification for $K=\mathbf{C}$ to obtain the description of all abstract overgroups of $G_{0}$ in $\operatorname{GL}\left(n^{2}, \mathbf{C}\right)$. The list of these overgroups is not as short as the one in [5], but it is not too long. The main result shows that any overgroup of $G_{0}$ in $\operatorname{GL}\left(n^{2}, \mathbf{C}\right)$ is "almost" a Lie subgroup. The deviation from Lie subgroups is expressed in terms of arbitrary subgroups of a 2-dimensional complex torus $T$, the centralizer of $G$ in $\mathrm{GL}\left(n^{2}, \mathbf{C}\right)$. In any case, our list of overgroups can be used effectively to solve many new linear preserver problems. We give here only one example, namely the linear preserver problem for normal matrices in $M_{n}$.

\section{Connected complex Lie overgroups of $\operatorname{PSL}(n, \mathbf{C})$}

Since the list of connected complex Lie overgroups of $G$ plays an important role in our proofs, we shall present this list below in Theorem A.

We have $M_{n}=M_{n}^{0} \oplus \mathbf{C} \cdot 1$ where $M_{n}^{0}$ is the subspace of $M_{n}$ defined by $\operatorname{tr}(x)=0$ and 1 denotes the identity matrix. The subgroup of $\operatorname{GL}\left(n^{2}, \mathbf{C}\right)$ which fixes 1 and leaves invariant $M_{n}^{0}$ will be denoted by $\mathrm{GL}\left(n^{2}-1, \mathbf{C}\right)$.

The symmetric bilinear form $\operatorname{tr}(x y)$ on $M_{n} \times M_{n}$ is non-degenerate. The subgroup of $\mathrm{GL}\left(n^{2}, \mathbf{C}\right)$ that preserves this form will be denoted by $\mathrm{O}\left(n^{2}, \mathbf{C}\right)$. $\mathrm{By} \mathrm{O}\left(n^{2}-1, \mathbf{C}\right)$ we denote its subgroup consisting of operators which fix 1 . As $M_{n}^{0} \perp \mathbf{C} \cdot 1$, we have $\mathrm{O}\left(n^{2}-1, \mathbf{C}\right)=\mathrm{O}\left(n^{2}, \mathbf{C}\right) \cap \mathrm{GL}\left(n^{2}-1, \mathbf{C}\right)$. We remark that if $n=2$, then $G=\mathrm{SO}\left(n^{2}-1, \mathbf{C}\right)$.

By $P($ resp. $Q)$ we denote the subgroup of $\operatorname{GL}\left(n^{2}, \mathbf{C}\right)$ which acts trivially on $M_{n}^{0}$ and $M_{n} / M_{n}^{0}$ (resp. C·1 and $M_{n} / \mathbf{C} \cdot 1$ ). By $T$ we denote the 2 -dimensional subtorus of $\operatorname{GL}\left(n^{2}, \mathbf{C}\right)$ which acts by scalar transformations on $M_{n}^{0}$ and $\mathbf{C} \cdot 1$. This torus centralizes $\operatorname{GL}\left(n^{2}-1, \mathbf{C}\right)$ and normalizes $P$ and $Q$. The center of $\operatorname{GL}\left(n^{2}, \mathbf{C}\right)$ will be denoted by $Z$. We also introduce the 1-dimensional torus $T_{1}=T \cap \operatorname{SL}\left(n^{2}, \mathbf{C}\right)$.

If we fix a basis of $M_{n}$ consisting of a basis of $M_{n}^{0}$ and the identity matrix 1, then $P, Q, T$ can be identified with the groups of all matrices of the form

$$
\left(\begin{array}{cc}
I_{n^{2}-1} & x \\
0 & 1
\end{array}\right), \quad\left(\begin{array}{cc}
I_{n^{2}-1} & 0 \\
y & 1
\end{array}\right), \quad\left(\begin{array}{cc}
\alpha I_{n^{2}-1} & 0 \\
0 & \beta
\end{array}\right)
$$

respectively, where $x$ is arbitrary column vector, $y$ arbitrary row vector, and $\alpha$ and $\beta$ arbitrary nonzero scalars.

Since $G$ consists of all operators $a \otimes a^{-1}$ with $a \in \mathrm{SL}(n, \mathbf{C})$, we have

$$
\mathrm{SL}(n, \mathbf{C}) \otimes \mathrm{SL}(n, \mathbf{C})^{\mathrm{opp}}=\{a \otimes b: a, b \in \mathrm{SL}(n, \mathbf{C})\} \supset G .
$$

We denote by $\tau$ the transposition map on $M_{n}$ and write $x^{\prime}$ instead of $\tau(x)$.

When $n=4$ we have constructed in [5] an overgroup $\Lambda \cong \mathrm{SL}(6, \mathbf{C}) /\langle-1\rangle$ of $G$. We recall the definition of $\Lambda$. Let $K_{4}$ be the space of skew-symmetric matrices in $M_{4}$ and $\operatorname{GL}(6, \mathbf{C})$ the group of invertible linear transformations of $K_{4}$. Define the 
homomorphism

$$
\theta: \mathrm{SL}(4, \mathbf{C}) \rightarrow \mathrm{SL}(6, \mathbf{C})
$$

by $\theta(a)(x)=a x a^{\prime}, x \in K_{4}$. We fix a basis of $K_{4}$ :

$$
\begin{array}{lll}
v_{1}=e_{12}-e_{21}, & v_{2}=e_{13}-e_{31}, & v_{3}=e_{14}-e_{41}, \\
v_{4}=e_{23}-e_{32}, & v_{5}=e_{24}-e_{42}, & v_{6}=e_{34}-e_{43},
\end{array}
$$

where $\left\{e_{i j}\right\}$ is the standard basis of $M_{4}$, and define the involution $f \in \mathrm{GL}(6, \mathbf{C})$ by

$$
\begin{array}{lll}
f\left(v_{1}\right)=v_{6}, & f\left(v_{2}\right)=-v_{5}, & f\left(v_{3}\right)=v_{4}, \\
f\left(v_{4}\right)=v_{3}, & f\left(v_{5}\right)=-v_{2}, & f\left(v_{6}\right)=v_{1} .
\end{array}
$$

It was shown in [5] that there is an isomorphism

$$
\varphi: K_{4} \wedge K_{4} \rightarrow M_{4}^{0}
$$

of $\mathrm{SL}(4, \mathbf{C})$-modules which is characterized by

$$
\varphi(x \wedge y)=x f(y)-y f(x), \quad x, y \in K_{4} .
$$

By transferring the natural action of $\operatorname{SL}(6, \mathbf{C})$ on $K_{4} \wedge K_{4}$ to $M_{4}^{0}$, via the isomorphism $\varphi$, we obtain a homomorphism

$$
\rho: \mathrm{SL}(6, \mathbf{C}) \rightarrow \mathrm{SL}(15, \mathbf{C}),
$$

with $\operatorname{Ker}(\rho)=\langle-1\rangle$. Explicitly we have

$$
\rho(a)(x f(y)-y f(x))=a(x) f(a(y))-a(y) f(a(x))
$$

for $x, y \in K_{4}$ and $a \in \operatorname{SL}(6, \mathbf{C})$. The group $\Lambda$ is the image of $\rho$, and $G$ is the image of $\rho \circ \theta$.

Theorem A A. Let $\Gamma$ be a proper connected complex Lie subgroup of $\operatorname{SL}\left(n^{2}, \mathbf{C}\right)$ containing $G=\operatorname{PSL}(n, \mathbf{C})$.

If $\Gamma$ is reducible, then $\Gamma=H, H P, H Q, H T_{1}, H P T_{1}$, or $H Q T_{1}$, where $H=G$, $\mathrm{SO}\left(n^{2}-1, \mathbf{C}\right), \mathrm{SL}\left(n^{2}-1, \mathbf{C}\right)$, or $\Lambda(n=4)$.

If $\Gamma$ is irreducible, then $\Gamma$ is a T-conjugate of $\mathrm{SL}(n, \mathbf{C}) \otimes \mathrm{SL}(n, \mathbf{C})^{\mathrm{opp}}$ or $\mathrm{SO}\left(n^{2}, \mathbf{C}\right)$.

Remark. In the case when $\Gamma$ is algebraic, this theorem is the complex version of [5, Theorem A]. For complex Lie subgroups $\Gamma$, the assertion of the theorem follows easily from the above special case.

\section{Connected compact overgroups of $\operatorname{PSU}(n) \operatorname{In} \operatorname{SL}\left(n^{2}, \mathbf{C}\right)$}

For the description of connected Lie overgroups of $G_{0}=\operatorname{PSU}(n)$ in $\operatorname{SL}\left(n^{2}, \mathbf{C}\right)$, we need some additional notations. The subgroup of $\mathrm{GL}\left(n^{2}, \mathbf{C}\right)$, which preserves the space of all hermitian matrices in $M_{n}$, will be denoted by $\operatorname{GL}\left(n^{2}, \mathbf{R}\right)$. By $x^{*}$ we denote the conjugate transpose of a matrix $x$. The involution $*$ on $M_{n}$ induces an involution, also denoted by $*$, on $M_{n} \otimes M_{n}^{\text {opp }}$. Namely for $z \in M_{n} \otimes M_{n}^{\text {opp }}$, viewed as a linear transformation on $M_{n}$, we define $z^{*}$ by :

$$
z^{*}(x)=\left(z\left(x^{*}\right)\right)^{*}, \quad x \in M_{n} .
$$

When $z=a \otimes b$ we have

$$
\begin{aligned}
(a \otimes b)^{*}(x) & =\left((a \otimes b)\left(x^{*}\right)\right)^{*}=\left(a x^{*} b\right)^{*}=b^{*} x a^{*} \\
& =\left(b^{*} \otimes a^{*}\right)(x), \quad x \in M_{n},
\end{aligned}
$$

i.e.,

$$
(a \otimes b)^{*}=b^{*} \otimes a^{*}
$$


Note that $z^{*}=z$ iff $z$ preserves the space of hermitian matrices in $M_{n}$. It follows that

$$
\operatorname{GL}\left(n^{2}, \mathbf{R}\right)=\left\{z \in \operatorname{GL}\left(n^{2}, \mathbf{C}\right): z^{*}=z\right\} .
$$

It is also straightforward to check that $G$ is $*$-invariant and that

$$
G_{0}=G \cap \operatorname{GL}\left(n^{2}, \mathbf{R}\right) .
$$

The subgroup of $\mathrm{GL}\left(n^{2}, \mathbf{C}\right)$ which preserves the positive definite hermitian form $\operatorname{tr}\left(x^{*} y\right)$ will be denoted by $\mathrm{U}\left(n^{2}\right)$. We also set

$$
\begin{aligned}
& \mathrm{SL}\left(n^{2}, \mathbf{R}\right)=\mathrm{SL}\left(n^{2}, \mathbf{C}\right) \cap \mathrm{GL}\left(n^{2}, \mathbf{R}\right), \\
& \mathrm{SU}\left(n^{2}\right)=\mathrm{SL}\left(n^{2}, \mathbf{C}\right) \cap \mathrm{U}\left(n^{2}\right), \\
& \mathrm{O}\left(n^{2}\right)=\mathrm{O}\left(n^{2}, \mathbf{C}\right) \cap \mathrm{GL}\left(n^{2}, \mathbf{R}\right), \\
& \mathrm{SO}\left(n^{2}\right)=\operatorname{SO}\left(n^{2}, \mathbf{C}\right) \cap \mathrm{GL}\left(n^{2}, \mathbf{R}\right), \\
& \mathrm{U}\left(n^{2}-1\right)=\mathrm{GL}\left(n^{2}-1, \mathbf{C}\right) \cap \mathrm{U}\left(n^{2}\right), \\
& \mathrm{SU}\left(n^{2}-1\right)=\mathrm{SL}\left(n^{2}-1, \mathbf{C}\right) \cap \mathrm{U}\left(n^{2}\right), \\
& \mathrm{O}\left(n^{2}-1\right)=\mathrm{O}\left(n^{2}-1, \mathbf{C}\right) \cap \mathrm{GL}\left(n^{2}, \mathbf{R}\right), \\
& \mathrm{SO}\left(n^{2}-1\right)=\mathrm{O}\left(n^{2}-1\right) \cap \mathrm{SL}\left(n^{2}, \mathbf{R}\right), \\
& P_{0}=P \cap \mathrm{GL}\left(n^{2}, \mathbf{R}\right), \\
& Q_{0}=Q \cap \mathrm{GL}\left(n^{2}, \mathbf{R}\right) .
\end{aligned}
$$

By $\mathrm{U}\left(n^{2}-1,1\right)$ we denote the subgroup of $\mathrm{GL}\left(n^{2}, \mathbf{C}\right)$, which preserves the nondegenerate hermitian form

$$
\operatorname{tr}\left(x^{*} y\right)-\operatorname{tr}\left(x^{*}\right) \operatorname{tr}(y)
$$

of Witt index 1. By $\mathrm{O}\left(n^{2}-1,1\right)$ we denote the subgroup of $\operatorname{GL}\left(n^{2}, \mathbf{R}\right)$ which preserves the non-degenerate symmetric bilinear form

$$
\operatorname{tr}(x y)-\operatorname{tr}(x) \operatorname{tr}(y)
$$

of Witt index 1 on the space of hermitian matrices in $M_{n}$. We set

$$
\begin{aligned}
& \mathrm{SU}\left(n^{2}-1,1\right)=\mathrm{U}\left(n^{2}-1,1\right) \cap \mathrm{SL}\left(n^{2}, \mathbf{C}\right), \\
& \mathrm{SO}\left(n^{2}-1,1\right)=\mathrm{O}\left(n^{2}-1,1\right) \cap \mathrm{SL}\left(n^{2}, \mathbf{R}\right) .
\end{aligned}
$$

We also introduce notation for two connected Lie subgroups of the torus $T_{1}$. Namely, $U_{1}$ will denote the circle group in $T_{1}$ and $R_{1}$ the subgroup of $T_{1}$ which acts as multiplication by positive scalars on $M_{n}^{0}$ (and $\mathbf{C} \cdot 1$ ).

When $n=4$, we introduce two real forms $\Lambda_{0}$ and $\Lambda_{1}$ of $\Lambda$. Let $\mathrm{U}(6)$ be the subgroup of $\mathrm{GL}(6, \mathbf{C})$ that preserves the positive definite hermitian form $\operatorname{tr}\left(x^{*} y\right)$ on $K_{4} \times K_{4}$. Then $\Lambda_{0} \cong \mathrm{SU}(6) /\langle-1\rangle$ is the image of $\mathrm{SU}(6)$ under the homomorphism $\rho$. The subgroup $\mathrm{SU}(4)$ of $\mathrm{SL}(4, \mathbf{C})$ perserves the above hermitian form, and consequently its image under the homomorphism $\theta$ is contained in $\mathrm{SU}(6)$. As the image of SU(4) under $\rho \circ \theta$ is $G_{0}$, we have $G_{0} \subset \Lambda_{0}$.

Let $\sigma: K_{4} \rightarrow K_{4}$ be the anti-linear involution defined by $\sigma(x)=f(\bar{x})=\overline{f(x)}$, where bar denotes complex conjugation. We have $\sigma(x)=x$ iff $f(x)=\bar{x}$. The map $\sigma$ induces on $\mathrm{SL}(6, \mathbf{C})$ an anti-holomorphic involution and provides this group with a structure of an algebraic group defined over $\mathbf{R}$. We shall denote by $\operatorname{SL}(6, \mathbf{R})$ its group of real points. Thus an element of $\operatorname{SL}(6, \mathbf{C})$ belongs to $\operatorname{SL}(6, \mathbf{R})$ iff it commutes with $\sigma$, or, equivalently, iff it preserves the real subspace of $K_{4}$ defined by $f(x)=\bar{x}$. We define $\Lambda_{1} \cong \mathrm{SL}(6, \mathbf{R}) /\langle-1\rangle$ to be the image of $\mathrm{SL}(6, \mathbf{R})$ under $\rho$. If $a \in \mathrm{GL}(6, \mathbf{C})$, i.e., $a$ is an invertible linear transformation on $K_{4}$, then we define

$$
a^{\sigma}:=\sigma \circ a \circ \sigma .
$$


Then $\operatorname{SL}(6, \mathbf{C})$ is $\sigma$-invariant and

$$
\mathrm{SL}(6, \mathbf{R})=\left\{a \in \mathrm{SL}(6, \mathbf{C}): a^{\sigma}=a\right\} .
$$

For $x \in K_{4}$ we have $x^{*}=-\bar{x}$. By using (1) and (2), we obtain

$$
\begin{aligned}
\rho(a)^{*}(x f(y)-y f(x)) & =\left[\rho(a)\left((x f(y)-y f(x))^{*}\right)\right]^{*} \\
& =[\rho(a)(f(\bar{y}) \bar{x}-f(\bar{x}) \bar{y})]^{*} \\
& =[\rho(a)(\sigma(y) f(\sigma(x))-\sigma(x) f(\sigma(y)))]^{*} \\
& =[a(\sigma(y)) f(a(\sigma(x)))-a(\sigma(x)) f(a(\sigma(y)))]^{*} \\
& =a^{\sigma}(x) f\left(a^{\sigma}(y)\right)-a^{\sigma}(y) f\left(a^{\sigma}(x)\right) \\
& =\rho\left(a^{\sigma}\right)(x f(y)-y f(x)) .
\end{aligned}
$$

Since this holds for all $x, y \in K_{4}$, we have

$$
\rho\left(a^{\sigma}\right)=\rho(a)^{*}, \quad a \in \mathrm{SL}(6, \mathbf{C}) .
$$

This implies that

$$
\Lambda_{1}=\Lambda \cap \mathrm{GL}\left(n^{2}, \mathbf{R}\right), \quad n=4 .
$$

Consequently $G_{0} \subset \Lambda_{1}$, and so $G_{0}$ is a maximal compact subgroup of $\Lambda_{1}$.

The connected compact overgroups of $G_{0}$ in $\mathrm{SL}\left(n^{2}, \mathbf{C}\right)$ are described in the next theorem.

Theorem 1. Let $\Gamma_{0}$ be a connected compact subgroup of $\mathrm{SL}\left(n^{2}, \mathbf{C}\right)$ containing $G_{0}$. If $\Gamma_{0}$ is reducible, then $\Gamma_{0}=H_{0}$ or $H_{0} U_{1}$ where

$$
H_{0}=G_{0}, \mathrm{SO}\left(n^{2}-1\right), \mathrm{SU}\left(n^{2}-1\right) \text {, or } \Lambda_{0} \text { if } n=4 .
$$

If $\Gamma_{0}$ is irreducible, then it is a T-conjugate of one of the groups:

$$
\mathrm{SO}\left(n^{2}\right), \mathrm{SU}(n) \otimes \mathrm{SU}(n)^{\mathrm{opp}}, \mathrm{SU}\left(n^{2}\right) .
$$

Proof. The complexification, $\Gamma$, of $\Gamma_{0}$ is either $\mathrm{SL}\left(n^{2}, \mathbf{C}\right)$ or one of the groups listed in Theorem A. If $\Gamma \neq \mathrm{SL}\left(n^{2}, \mathbf{C}\right)$, we claim that $\Gamma_{0}$ is the unique maximal compact subgroup of $\Gamma$ containing $G_{0}$.

In order to prove this claim, we start by choosing a positive definite $\Gamma_{0}$-invariant hermitian form $h: M_{n} \times M_{n} \rightarrow \mathbf{C}$. As $M_{n}^{0}$ is an irreducible $G_{0}$-module, there is only one (up to a scalar factor) positive definite $G_{0}$-invariant hermitian form on $M_{n}^{0} \times M_{n}^{0}$. Therefore we may assume that

$$
h(x, y)=\operatorname{tr}\left(x^{*} y\right), \quad x, y \in M_{n}^{0} .
$$

If $\Gamma=H$ (see Theorem $\mathrm{A}$ ) is simple and reducible, it follows that $\Gamma_{0}=\Gamma \cap$ $\mathrm{SU}\left(n^{2}-1\right)$. Thus our claim holds in that case. Clearly, it also holds if $\Gamma=H T_{1}$.

If $\Gamma$ is irreducible, then by using Theorem A we see that it suffices to prove our claim when $\Gamma$ is $\mathrm{SO}\left(n^{2}, \mathbf{C}\right)$ or $\mathrm{SL}(n, \mathbf{C}) \otimes \mathrm{SL}(n, \mathbf{C})^{\text {opp }}$. We consider these two cases separately.

Case 1. $\Gamma=\mathrm{SO}\left(n^{2}, \mathbf{C}\right)$. There is a unique anti-linear automorphism $u: M_{n} \rightarrow M_{n}$ such that $h(x, y)=\operatorname{tr}(u(x) y)$ for all $x, y \in M_{n}$. For $x, y \in M_{n}^{0}$, we have

$$
\operatorname{tr}\left(x^{*} y\right)=h(x, y)=\operatorname{tr}(u(x) y)
$$

i.e., $\operatorname{tr}\left(\left(u(x)-x^{*}\right) y\right)=0$. Consequently, $u(x)=x^{*}+\psi(x) \cdot 1$ for some anti-linear function $\psi: M_{n}^{0} \rightarrow \mathbf{C}$. As $h(x, y)$ and $\operatorname{tr}(x y)$ are both $\Gamma_{0}$-invariant, $u$ commutes 
with the action of $\Gamma_{0}$, i.e., we have $u\left(a x a^{-1}\right)=a u(x) a^{-1}$ for all $a \in \operatorname{SU}(n)$ and $x \in M_{n}$. This implies that $\psi\left(a x a^{-1}\right)=\psi(x)$ for all $x \in M_{n}^{0}$ and $a \in \operatorname{SU}(n)$. Thus $\psi$ is a homomorphism of $\mathrm{SU}(n)$-modules, and so $\psi=0$. Hence $u(x)=x^{*}$ for all $x \in M_{n}^{0}$.

For $x \in M_{n}^{0}, h(x, 1)=\operatorname{tr}(u(x))=\operatorname{tr}\left(x^{*}\right)=0$ and $\operatorname{tr}(u(1) x)=h(1, x)=0$. This implies that $u(1)=\lambda \cdot 1$ for some $\lambda \in \mathbf{C}^{*}$. As $n \lambda=\operatorname{tr}(u(1))=h(1,1)>0$, we have $\lambda>0$.

The restrictions of $\operatorname{tr}(x y)$ and $\operatorname{tr}\left(x^{*} y\right)$ to the space of Hermitian $n$ by $n$ matrices of trace 0 coincide. We choose an orthonormal basis $B_{0}$ for this space. Then $B_{0}$ together with $(1 / \sqrt{n}) \cdot 1$ provides an orthonormal basis $B$ for the form $\operatorname{tr}(x y)$. The matrix of $\operatorname{tr}(x y)$ with respect to $B$ is the identity matrix while that of $h$ is

$$
J=\left(\begin{array}{cc}
I_{n^{2}-1} & 0 \\
0 & \lambda
\end{array}\right) .
$$

With respect to this basis, $\mathrm{SO}\left(n^{2}, \mathbf{C}\right)$ consists of all complex matrices $x$ satisfying $x x^{\prime}=I_{n^{2}}$, and $\mathrm{SU}(h)$ consists of all complex matrices $x$ satisfying $x^{*} J x=J$. The matrices $x \in \Gamma_{0}$ satisfy both of these conditions, and so $\bar{x} J=J x$. Since $\Gamma_{0}$ is irreducible, the last condition easily implies that $\lambda=1$. Consequently $\Gamma_{0}=\mathrm{SO}\left(n^{2}\right)$.

Case 2. $\Gamma=\mathrm{SL}(n, \mathbf{C}) \otimes \mathrm{SL}(n, \mathbf{C})^{\mathrm{opp}}$. In this case we must have $\Gamma_{0}=A \otimes B$ where $A$ and $B$ are maximal compact subgroups of $\mathrm{SL}(n, \mathbf{C})$, i.e., they are conjugates of $\mathrm{SU}(n)$. Since

$$
\Gamma_{0} \supset G_{0}=\left\{a \otimes a^{-1}: a \in \mathrm{SU}(n)\right\},
$$

it follows that $A=B=\mathrm{SU}(n)$. This completes the proof of our claim.

It remains to prove the assertion of the theorem in the case $\Gamma=\operatorname{SL}\left(n^{2}, \mathbf{C}\right)$. As above, we find that $\Gamma_{0}$ preserves a positive definite Hermitian form $h(x, y)$ such that $h(x, y)=\operatorname{tr}\left(x^{*} y\right)$ for $x, y \in M_{n}^{0}$. As $h$ is also $G_{0}$-invariant, the $h$-orthogonal complement of $M_{n}^{0}$ in $M_{n}$ is a $G_{0}$-submodule. Hence this orthogonal complement must be $\mathbf{C} \cdot 1$. Let $\lambda:=h(1,1)>0$ and $\mu=\sqrt{\lambda / n}$. Define $t \in T$ by $t(x)=x$ for all $x \in M_{n}^{0}$ and $t(1)=\mu \cdot 1$. Then $h(x, y)=\operatorname{tr}\left(t(x)^{*} t(y)\right)$ holds for all $x, y \in M_{n}$. It follows that $\Gamma_{0}=t^{-1} \cdot \mathrm{SU}\left(n^{2}\right) \cdot t$.

\section{Abstract overgroups of $\operatorname{PSU}(n)$ in $\operatorname{GL}\left(n^{2}, \mathbf{C}\right)$}

We first describe all connected Lie overgroups of $G_{0}$ in $\operatorname{SL}\left(n^{2}, \mathbf{C}\right)$. For that purpose we need the following two lemmas.

Lemma 1. Let $\mathfrak{g}$ be a finite-dimensional semisimple complex Lie algebra and $\mathfrak{g}_{0}$ a real Lie subalgebra of $\mathfrak{g}$ such that $\mathfrak{g}=\mathfrak{g}_{0}+i \mathfrak{g}_{0}$. Then $\mathfrak{a}=\mathfrak{g}_{0} \cap i \mathfrak{g}_{0}$ is an ideal of $\mathfrak{g}$, $\mathfrak{g}=\mathfrak{a} \times \mathfrak{b}$, and $\mathfrak{g}_{0}=\mathfrak{a} \times \mathfrak{b}_{0}$, where $\mathfrak{b}_{0}$ is a real form of $\mathfrak{b}$.

Proof. It is clear that $\mathfrak{a}$ is a complex subspace of $\mathfrak{g}$. It is also evident that $\mathfrak{a}$ is an ideal of $\mathfrak{g}_{0}$. Since $\mathfrak{a}=\mathfrak{g}_{0}+i \mathfrak{g}_{0}$ and

$$
\left[\mathfrak{a}, i \mathfrak{g}_{0}\right]=\left[i \mathfrak{a}, \mathfrak{g}_{0}\right]=\left[\mathfrak{a}, \mathfrak{g}_{0}\right] \subset \mathfrak{a},
$$

it follows that $\mathfrak{a}$ is an ideal of $\mathfrak{g}$. Hence $\mathfrak{g}=\mathfrak{a} \times \mathfrak{b}$ for some ideal $\mathfrak{b}$ of $\mathfrak{g}$. As $\mathfrak{g}_{0} \supset \mathfrak{a}$, we have $\mathfrak{g}_{0}=\mathfrak{a} \times \mathfrak{b}_{0}$ for some real subalgebra $\mathfrak{b}_{0}$ of $\mathfrak{b}$. Since $\mathfrak{b}_{0}+i \mathfrak{b}_{0}=\mathfrak{b}$ and $\mathfrak{b}_{0} \cap i \mathfrak{b}_{0}=0, \mathfrak{b}_{0}$ is a real form of $\mathfrak{b}$.

Lemma 2. Let $\mathfrak{g}$ be a finite-dimensional simple complex Lie algebra and $\mathfrak{g}_{1}, \mathfrak{g}_{2}$ two isomorphic real forms of $\mathfrak{g}$. If $\mathfrak{g}_{1} \cap \mathfrak{g}_{2}$ contains a maximal compact Lie subalgebra $\mathfrak{k}$ of $\mathfrak{g}_{1}$ and $\mathfrak{k}$ is semisimple, then $\mathfrak{g}_{1}=\mathfrak{g}_{2}$. 
Proof. Let $\mathfrak{g}_{1}=\mathfrak{k} \oplus \mathfrak{p}_{1}$ and $\mathfrak{g}_{2}=\mathfrak{k} \oplus \mathfrak{p}_{2}$ be Cartan decompositions. Then $\mathfrak{p}_{1} \perp \mathfrak{k}$ and $\mathfrak{p}_{2} \perp \mathfrak{k}$ with respect to the Killing form of $\mathfrak{g}$. Consequently $\mathfrak{p}_{2} \subset \mathfrak{p}:=\mathfrak{p}_{1}+i \mathfrak{p}_{1}$. As $\mathfrak{g}_{1}$ is absolutely simple and $\mathfrak{k}$ is semisimple, $\mathfrak{p}_{1}$ is an absolutely simple $\mathfrak{k}$-module, see $[2$, Proposition (8.8.3)]. This implies that $\mathfrak{p}_{2}=\alpha \mathfrak{p}_{1}$ for some complex number $\alpha$ with $|\alpha|=1$. As $\left[\mathfrak{p}_{2}, \mathfrak{p}_{2}\right] \subset \mathfrak{k}$, we have $\alpha^{2} \in \mathbf{R}$, and so $\mathfrak{p}_{2}=\mathfrak{p}_{1}$ or $\mathfrak{p}_{2}=i \mathfrak{p}_{1}$. Since $\mathfrak{k}+i \mathfrak{p}_{1}$ is a compact real form of $\mathfrak{g}$ and $\mathfrak{g}_{1} \cong \mathfrak{g}_{2}$, we must have $\mathfrak{p}_{2}=\mathfrak{p}_{1}$, i.e., $\mathfrak{g}_{2}=\mathfrak{g}_{1}$.

Theorem 2. Let $\Gamma$ be a proper connected Lie subgroup of $\mathrm{SL}\left(n^{2}, \mathbf{C}\right)$ containing $G_{0}$. If $\Gamma$ is reducible, then either

$$
\Gamma=H R, H P R, H Q R,
$$

where

$$
\begin{aligned}
H= & G_{0}, G, \mathrm{SO}\left(n^{2}-1\right), \mathrm{SO}\left(n^{2}-1, \mathbf{C}\right), \mathrm{SU}\left(n^{2}-1\right), \\
& \mathrm{SL}\left(n^{2}-1, \mathbf{R}\right), \mathrm{SL}\left(n^{2}-1, \mathbf{C}\right), \text { or } \Lambda_{0}, \Lambda_{1}, \Lambda(n=4),
\end{aligned}
$$

and $R$ is a connected Lie subgroup of $T_{1}$ (which may be trivial), or $\Gamma$ is a $U_{1}$ conjugate of one of the groups :

$$
H P_{0}, H Q_{0}, H P_{0} R_{1}, H Q_{0} R_{1}
$$

where

$$
H=G_{0}, \mathrm{SO}\left(n^{2}-1\right), \mathrm{SL}\left(n^{2}-1, \mathbf{R}\right) \text {, or } \Lambda_{0}, \Lambda_{1}(n=4) .
$$

If $\Gamma$ is irreducible, then $\Gamma$ is a T-conjugate of one of the groups:

$$
\begin{aligned}
& \mathrm{SL}\left(n^{2}, \mathbf{R}\right), \mathrm{SU}\left(n^{2}\right), \mathrm{SU}\left(n^{2}-1,1\right), \\
& \mathrm{SO}\left(n^{2}, \mathbf{C}\right), \mathrm{SO}\left(n^{2}\right), \mathrm{SO}\left(n^{2}-1,1\right)^{0}, \\
& \mathrm{SL}(n, \mathbf{C}) \otimes \mathrm{SL}(n, \mathbf{C})^{\mathrm{opp}}, \mathrm{SU}(n) \otimes \mathrm{SL}(n, \mathbf{C})^{\mathrm{opp}}, \\
& \mathrm{SL}(n, \mathbf{C}) \otimes \mathrm{SU}(n)^{\mathrm{opp}}, \mathrm{SU}(n) \otimes \mathrm{SU}(n)^{\text {opp }}, \\
& \left\{a \otimes a^{*}: a \in \operatorname{SL}(n, \mathbf{C})\right\} .
\end{aligned}
$$

Proof. In view of Theorem A and Theorem 1, we may assume that $\Gamma$ is neither a complex Lie group nor a compact group. Let $L \subset \mathrm{SL}\left(n^{2}, \mathbf{C}\right)$ be the smallest complex Lie subgroup containing $\Gamma$, and $\Gamma_{0} \supset G_{0}$ a maximal compact subgroup of $\Gamma$. Then $L$ is either $\operatorname{SL}\left(n^{2}, \mathbf{C}\right)$ or one of the groups listed in Theorem A. We shall consider the various possibilities for $L$ separately. If $L$ is reducible and semisimple, there are four cases to consider.

Case 1. $L=\mathrm{SL}\left(n^{2}-1, \mathbf{C}\right)$. By Lemma $1, \Gamma$ is a real form of $L$. For the list of real forms of almost simple complex Lie groups see [4]. By Theorem $1, \Gamma_{0}=G_{0}$, $\mathrm{SO}\left(n^{2}-1\right)$, or $\Lambda_{0}$ if $n=4$. Hence $\Gamma$ is not isomorphic to $\mathrm{SU}(p, q)$ for $p+q=n^{2}-1$, $p \geq q \geq 1$. If $\Gamma \cong \operatorname{SL}\left(n^{2}-1, \mathbf{R}\right)$, then Lemma 2 implies that $\Gamma=\operatorname{SL}\left(n^{2}-1, \mathbf{R}\right)$. The real form $\mathrm{SL}\left(\left(n^{2}-1\right) / 2, \mathbf{H}\right), n$ odd, is ruled out because its maximal compact subgroup is $\operatorname{Sp}\left(\left(n^{2}-1\right) / 2\right)$.

Case 2. $L=\mathrm{SO}\left(n^{2}-1, \mathbf{C}\right)$. By Lemma $1, \Gamma$ is a real form of $L$. By Theorem 1 , $\Gamma_{0}=G_{0}$ or $\Lambda_{0}$ if $n=4$. The real forms $\mathrm{SO}(p, q)^{0}, p+q=n^{2}-1, p \geq q \geq 1$, are ruled out because their maximal compact subgroups are not isomorphic to $G_{0}$ or $\Lambda_{0}$ if $n=4$. The real form $\mathrm{SO}^{*}\left(n^{2}-1\right), n$ odd, is also ruled out by the same argument.

Case 3. $L=G$. By Lemma 1, $\Gamma$ must be a real form of $G$. Since $\Gamma \supset G_{0}$ and we assume that $\Gamma$ is not compact, this case cannot occur. 
Case 4. $L=\Lambda(n=4)$. By Lemma $1, \Gamma$ is a real form of $\Lambda$. Hence $\Gamma$ is isomorphic to $\mathrm{SU}(p, q) /\langle-1\rangle, p+q=6, p \geq q \geq 1$, $\mathrm{SL}(3, \mathbf{H}) /\langle-1\rangle$, or $\Lambda_{1}$. By inspecting the maximal compact subgroups of these real forms we see that $\Gamma \cong \Lambda_{1}$, and so $\Gamma=\Lambda_{1}$ by Lemma 2 .

Next we assume that $L$ is reducible and reductive, but not semisimple. Then the maximal semisimple subgroup $H$ of $\Gamma$ contains $G_{0}$. By taking into account the previous cases, we conclude that $H$ is one of the groups listed in (6). Since $T_{1}$ is the centralizer of $G_{0}$ in $\operatorname{SL}\left(n^{2}, \mathbf{C}\right)$, we have $\Gamma=H R$ where $R$ is a non-trivial connected Lie subgroup of $T_{1}$.

When $L$ is irreducible, there are three cases to consider.

Case 5. $L=\operatorname{SL}\left(n^{2}, \mathbf{C}\right)$. By Lemma $1, \Gamma$ is a real form of $L$. Assume first that $\Gamma \cong \operatorname{SL}\left(n^{2}, \mathbf{R}\right)$. Then, by Theorem $1, \Gamma_{0}=t \cdot \mathrm{SO}\left(n^{2}\right) \cdot t^{-1}$ for some $t \in T$. By Lemma 2 we have $\Gamma=t \cdot \operatorname{SL}\left(n^{2}, \mathbf{R}\right) \cdot t^{-1}$. Next assume that $\Gamma \cong \mathrm{SU}(p, q)$ where $p+q=n^{2}, p \geq q \geq 1$. Theorem 1 implies that $p=n^{2}-1$ and $q=1$. By the same argument as above, we conclude that $\Gamma=t \cdot \mathrm{SU}\left(n^{2}-1,1\right) \cdot t^{-1}$ for some $t \in T$. The real form $\mathrm{SL}\left(n^{2} / 2, \mathbf{H}\right), n$ even, is ruled out because its maximal compact subgroup $\operatorname{Sp}\left(n^{2} / 2\right)$ does not occur in Theorem 1 .

Case 6. $L=t \cdot \mathrm{SO}\left(n^{2}, \mathbf{C}\right) \cdot t^{-1}, t \in T$. Without any loss of generality, we may assume that $t=1$. By Lemma $1, \Gamma$ is isomorphic to $\mathrm{SO}(p, q)^{0}, p+q=n^{2}, p \geq q \geq 1$, or $\mathrm{SO}^{*}\left(n^{2}\right), n$ even. The latter case is ruled out because a maximal compact subgroup of $\mathrm{SO}^{*}\left(n^{2}\right)$ is isomorphic to $\mathrm{U}\left(n^{2} / 2\right)$. In the former case, Theorem 1 implies that $p=n^{2}-1, q=1$, and that $\Gamma_{0}=\mathrm{SO}\left(n^{2}-1\right)$. By Lemma 2, we conclude that $\Gamma=\mathrm{SO}\left(n^{2}-1,1\right)^{0}$.

Case 7. $L=t \cdot\left(\mathrm{SL}(n \mathbf{C}) \otimes \mathrm{SL}(n, \mathbf{C})^{\mathrm{opp}}\right) \cdot t^{-1}, t \in T_{1}$. Again we may assume that $t=1$. Since

$$
\Gamma \supset G_{0}=\left\{a \otimes a^{-1}: a \in \mathrm{SU}(n)\right\},
$$

Lemma 1 implies that $\Gamma$ is either one of the groups

$$
\mathrm{SL}(n, \mathbf{C}) \otimes \mathrm{SU}(n)^{\mathrm{opp}}, \mathrm{SU}(n) \otimes \mathrm{SL}(n, \mathbf{C})^{\mathrm{opp}},
$$

or a real form of $L$. In the latter case $\Gamma$ is either $\mathrm{SU}(n) \otimes \mathrm{SU}(n)^{\text {opp }}$ or the group $\left\{a \otimes a^{*}: a \in \mathrm{SL}(n, \mathbf{C})\right\} \cong \mathrm{PSL}_{n}(\mathbf{C})$.

It remains to consider the cases where $L$ is not reductive. By Theorem $\mathrm{A}, L$ is reducible and has the form $H P, H Q, H P T_{1}, H Q T_{1}$ with $H=G, \mathrm{SO}\left(n^{2}-1, \mathbf{C}\right)$, $\mathrm{SL}\left(n^{2}-1, \mathbf{C}\right)$ or $\Lambda$ if $n=4$.

If $\Gamma \supset P($ or $Q$ ), then $\Gamma$ is a semidirect product of $P$ (or $Q$ ) and some reducible reductive group $F \supset G_{0}$. We have shown that $F=H R$ where $H$ is one of the groups in (6) and $R$ is a connected Lie subgroup of $T_{1}$. Hence $\Gamma$ is one of the groups in (5).

Let $\mathfrak{p}\left(\right.$ resp $\left.\mathfrak{p}_{0}\right)$ be the Lie algebra of $P\left(\right.$ resp. $\left.P_{0}\right)$. Then $\mathfrak{p}=\mathfrak{p}_{0} \oplus i \mathfrak{p}_{0}$ and $\mathfrak{p}_{0}$ is a simple $G_{0}$-module. Furthermore every simple $G_{0}$-submodule of $\mathfrak{p}$ has the form $\alpha \mathfrak{p}_{0}$ for some $\alpha \in \mathbf{C}^{*}$, and so it is a $U_{1}$-conjugate of $\mathfrak{p}_{0}$.

Assume that $L \supset P$ but $\Gamma \not \supset P$. Let $\mathfrak{r}$ be the solvable radical of the Lie algebra of $\Gamma$. If $\mathfrak{t}_{1}$ is the Lie algebra of the torus $T_{1}$, then $\mathfrak{r} \subset \mathfrak{p}+\mathfrak{t}_{1}$. As $\mathfrak{t}_{1}$ is a trivial $G_{0}$-module, we must have $\mathfrak{r}=(\mathfrak{r} \cap \mathfrak{p}) \oplus\left(\mathfrak{r} \cap \mathfrak{t}_{1}\right)$. As $L \supset P$, we have $\mathfrak{r} \cap \mathfrak{p} \neq 0$. As $\Gamma \not \supset P$, we have $\mathfrak{r} \cap \mathfrak{p} \neq \mathfrak{p}$. Therefore $\mathfrak{r} \cap \mathfrak{p}=\alpha \mathfrak{p}_{0}$ for some $\alpha \in \mathbf{C}^{*}$. By replacing $\Gamma$ with a suitable $U_{1}$-conjugate, we can assume that $\mathfrak{r} \cap \mathfrak{p}=\mathfrak{p}_{0}$. It follows that $\Gamma \cap P=P_{0}$ and that $\Gamma$ is a semidirect product of $P_{0}$ and a reducible reductive 
group $F \supset G_{0}$. By using the description of such $F$, we conclude that $\Gamma$ is one of the groups in (7).

Corollary 1. Let $\Gamma$ be a proper connected Lie subgroup of $\mathrm{GL}\left(n^{2}, \mathbf{C}\right)$ containing $G_{0}$. If $\Gamma$ is reducible, $\Gamma$ is either one of the groups (5) where $R$ is now a connected Lie subgroup of $T$, or a $U_{1}$-conjugate of one of the groups $H P_{0} R, H Q_{0} R$, where $H$ is as in (8) and $R$ is a connected Lie subgroup of $R_{1} Z$. If $\Gamma$ is irreducible, then $\Gamma=H R$ where $H$ is $\mathrm{SL}\left(n^{2}, \mathbf{C}\right)$ or a $T$-conjugate of a group in (9) and $R$ is a connected Lie subgroup of $Z$.

For applications, in particular to linear preserver problems, it is important to have a description of all (abstract) overgroups of $G_{0}$ in $\operatorname{GL}\left(n^{2}, \mathbf{C}\right)$. Let $L$ be a Lie group and $\Gamma$ an abstract subgroup of $L$. Denote by $\Sigma$ the collection of all connected Lie subgroups of $L$ which are contained in $\Gamma$. Since arcwise connected subgroups of $L$ are Lie subgroups [3], $\Sigma$ has a unique largest element, say $\Gamma^{0}$. Thus $\Gamma^{0}$ contains all subgroups in $\Sigma$. By construction, $\Gamma^{0}$ is a normal subgroup of $\Gamma$. Consequently we have the following theorem.

Theorem 3. Let $\Gamma$ be an abstract subgroup of $\operatorname{GL}\left(n^{2}, \mathbf{C}\right)$ containing $G_{0}$, and let $\Gamma^{0}$ be the largest connected Lie subgroup contained in $\Gamma$. Then

$$
G_{0} \subset \Gamma^{0} \subset \Gamma \subset N\left(\Gamma^{0}\right),
$$

where $N\left(\Gamma^{0}\right)$ is the normalizer of $\Gamma^{0}$ in $\mathrm{GL}\left(n^{2}, \mathbf{C}\right)$.

Now the description of all overgroups of $G_{0}$ is reduced to the computation of the normalizers of all possible connected Lie overgroups of $G_{0}$.

Theorem 4. Let $\Gamma$ be a connected Lie subgroup of $\mathrm{GL}\left(n^{2}, \mathbf{C}\right)$ containing $G_{0}$, and let $N(\Gamma)$ be the normalizer of $\Gamma$ in $\mathrm{GL}\left(n^{2}, \mathbf{C}\right)$. As in Corollary 1, let $H$ be a maximal semisimple subgroup of $\Gamma$ containing $G_{0}$. Then

(i) $N(\Gamma)=\Gamma T$ if $H$ is one of the groups $\mathrm{SL}\left(n^{2}-1, \mathbf{C}\right), \mathrm{SU}\left(n^{2}-1\right)$, or $\Lambda, \Lambda_{0}$ $(n=4)$;

(ii) $N(\Gamma)=\Gamma T\langle\tau\rangle$ if $H$ is one of the groups $G, G_{0}$, or $\Lambda_{1}(n=4)$;

(iii) $N(\Gamma)=\mathrm{O}\left(n^{2}-1\right) \Gamma T$ if $H$ is one of the groups $\mathrm{SO}\left(n^{2}-1, \mathbf{C}\right), \mathrm{SO}\left(n^{2}-1\right)$, or $\mathrm{SL}\left(n^{2}-1, \mathbf{R}\right)$;

(iv) $N(\Gamma)=\Gamma Z$ if $H$ is one of the groups $\mathrm{SL}\left(n^{2}, \mathbf{C}\right), \mathrm{SU}\left(n^{2}\right), \mathrm{SU}\left(n^{2}-1,1\right), \mathrm{SU}(n)$ $\otimes \mathrm{SL}(n, \mathbf{C})^{\mathrm{opp}}$, or $\mathrm{SL}(n, \mathbf{C}) \otimes \mathrm{SU}(n)^{\mathrm{opp}}$;

(v) $N(\Gamma)=\Gamma Z\langle\tau\rangle$ if $H$ is one of the groups $\mathrm{SL}(n, \mathbf{C}) \otimes \mathrm{SL}(n, \mathbf{C})^{\text {opp }}, \mathrm{SU}(n) \otimes$ $\mathrm{SU}(n)^{\text {opp }}$, or $\left\{a \otimes a^{*}: a \in \mathrm{SL}(n, \mathbf{C})\right\}$;

(vi) $N(\Gamma)=\mathrm{O}\left(n^{2}-1\right) \Gamma Z$ if $H$ is one of the groups $\mathrm{SO}\left(n^{2}, \mathbf{C}\right), \mathrm{SO}\left(n^{2}\right)$, $\mathrm{SO}\left(n^{2}-1,1\right)^{0}$, or $\mathrm{SL}\left(n^{2}, \mathbf{R}\right)$.

Proof. All normalizers will be taken in $\mathrm{GL}\left(n^{2}, \mathbf{C}\right)$. As the maximal semisimple subgroups of $\Gamma$ are all conjugate in $\Gamma$, we have $N(\Gamma) \subset \Gamma N(H)$. Let $H_{0}$ be a maximal compact subgroup of $H$ containing $G_{0}$. As the maximal compact subgroups of $H$ are all conjugate in $H$, we have $N(H) \subset H N\left(H_{0}\right)$. Hence

$$
N(\Gamma) \subset \Gamma N\left(H_{0}\right)
$$


Let $L \subset \mathrm{SL}\left(n^{2}, \mathbf{C}\right)$ be the complexification of $H_{0}$. By [5, Theorem B], we have

$$
N(L)= \begin{cases}G T\langle\tau\rangle & \text { if } L=G, \\ L T & \text { if } L=\mathrm{SL}\left(n^{2}-1, \mathbf{C}\right) \text { or } \Lambda(n=4), \\ \mathrm{O}\left(n^{2}-1, \mathbf{C}\right) T & \text { if } L=\mathrm{SO}\left(n^{2}-1, \mathbf{C}\right), \\ \mathrm{O}\left(n^{2}, \mathbf{C}\right) Z & \text { if } L=\mathrm{SO}\left(n^{2}, \mathbf{C}\right), \\ L Z\langle\tau\rangle & \text { if } L=\mathrm{SL}(n, \mathbf{C}) \otimes \mathrm{SL}(n, \mathbf{C})^{\text {opp }}\end{cases}
$$

Since $H_{0}$ is its own normalizer in $H$, see [4, p. 275], and $\tau$ normalizes $G_{0}$ and $\mathrm{SU}(n) \otimes \mathrm{SU}(n)^{\mathrm{opp}}$, it follows easily that

$$
N\left(H_{0}\right)= \begin{cases}G_{0} T\langle\tau\rangle & \text { if } H_{0}=G_{0} \\ H_{0} T & \text { if } H_{0}=\mathrm{SU}\left(n^{2}-1\right) \text { or } \Lambda_{0}(n=4), \\ \mathrm{O}\left(n^{2}-1\right) T & \text { if } H_{0}=\mathrm{SO}\left(n^{2}-1\right), \\ \mathrm{O}\left(n^{2}\right) Z & \text { if } H_{0}=\mathrm{SO}\left(n^{2}\right) \\ H_{0} Z\langle\tau\rangle & \text { if } H_{0}=\mathrm{SU}(n) \otimes \mathrm{SU}(n)^{\mathrm{opp}} \\ H_{0} Z & \text { if } H_{0}=\mathrm{SU}\left(n^{2}\right) .\end{cases}
$$

We can now determine $N(\Gamma)$ by using (10), Theorem 2 and Corollary 1. For each $H_{0}$ and all possible $H$ 's and $\Gamma$ 's, it is easy to check that the identity component of $N\left(H_{0}\right)$ normalizes $H$ and $\Gamma$. We shall now consider the cases where $N\left(H_{0}\right)$ is not connected.

If $H_{0}=G_{0}$, then $H$ is one of the groups $G_{0}, G, \Lambda_{1}(n=4)$, or $\left\{a \otimes a^{*}: a \in\right.$ $\operatorname{SL}(n, \mathbf{C})\}$. If $n=4, \tau$ normalizes $\Lambda$, see [5], and we have shown in Section 5 that $\Lambda_{1}=\Lambda \cap \mathrm{GL}\left(n^{2}, \mathbf{R}\right)$. It follows that $\tau$ normalizes $\Lambda$. Thus in all cases $\tau$ normalizes $H$ (and $\Gamma$ ).

If $H_{0}=\mathrm{SO}\left(n^{2}-1\right)$, then $H$ is one of the groups $H_{0}, \mathrm{SL}\left(n^{2}-1, \mathbf{R}\right), \mathrm{SO}\left(n^{2}-1, \mathbf{C}\right)$, or $\mathrm{SO}\left(n^{2}-1,1\right)^{0}$. In all these cases $\mathrm{O}\left(n^{2}-1\right)$ normalizes $H$ (and $\Gamma$ ).

If $H_{0}=\mathrm{SO}\left(n^{2}\right)$, then either $H=H_{0}$ or $\mathrm{SO}\left(n^{2}, \mathbf{C}\right)$. In both cases $\mathrm{O}\left(n^{2}\right)$ normalizes $H$ (and $\Gamma$ ).

Finally if $H_{0}=\mathrm{SU}(n) \otimes \mathrm{SU}(n)^{\mathrm{opp}}$, then $H$ is one of the groups $H_{0}, \mathrm{SL}(n, \mathbf{C}) \otimes$ $\mathrm{SL}(n, \mathbf{C})^{\text {opp }}, \mathrm{SU}(n) \otimes \mathrm{SL}(n, \mathbf{C})^{\text {opp }}$, or $\mathrm{SL}(n, \mathbf{C}) \otimes \mathrm{SU}(n)^{\text {opp }}$. Since $\tau \circ(a \otimes b) \circ \tau=$ $b^{\prime} \otimes a^{\prime}$, only the first two of these groups are normalized by $\tau$.

In view of (10), this completes the proof of the theorem.

This theorem shows that $N(\Gamma)$ is not much larger than $\Gamma$. For instance we have $N(\Gamma)^{0}=\Gamma T$ or $\Gamma Z$, and $\left[N(\Gamma): N(\Gamma)^{0}\right]$ is 1 or 2 .

\section{LiNEAR PRESERVER PROBLEM FOR NORMAL MATRICES}

Let $X \subset M_{n}$ be the set of normal matrices, i.e., matrices $x$ satisfying $x x^{*}=x^{*} x$. Since $X$ is a (real) algebraic set, the subset

$$
G_{X}:=\left\{a \in \operatorname{GL}\left(n^{2}, \mathbf{C}\right): a(X) \subset X\right\}
$$

is in fact a subgroup of $\operatorname{GL}\left(n^{2}, \mathbf{C}\right)$, see [5]. It is also evident that $G_{X}$ is closed and $G_{X} \supset G_{0}$. We shall illustrate our method for solving a wide class of linear preserver problems by applying it to determine the group $G_{X}$. The groups $Q, T$ and the element $\tau$, used in the next theorem, have been defined in previous sections. 
Theorem 5. If $X \subset M_{n}$ is the set of normal matrices, then $G_{X}=H Q T\langle\tau\rangle$ where $H=G_{0}$ for $n>2$ and $H=\operatorname{SL}(3, \mathbf{R})$ for $n=2$. If $n>2$, this means that any nonsingular linear transformation $u$ of $M_{n}$ such that $u(X) \subset X$ has the form

$$
u(x)=\alpha a x a^{-1}+\psi(x) \cdot 1,
$$

where $\alpha \in \mathbf{C}^{*}, a \in U(n)$, and $\psi: M_{n} \rightarrow \mathbf{C}$ is a linear function.

Proof. Let $u \in G_{X}$ and $a=u(1)$ where 1 is the identity matrix. Since $1+X=X$ and $u(X)=X$, we have $a+X=X$. Hence

$$
(a+x)(a+x)^{*}=(a+x)^{*}(a+x), \quad x \in X,
$$

i.e., $a x^{*}-x^{*} a=a^{*} x-x a^{*}$ for all $x \in X$. By replacing $x$ with $i x$, we infer that $a x^{*}=x^{*} a$ for all $x \in X$. As $X$ spans $M_{n}$, we conclude that $a$ is a central element of $M_{n}$, i.e., $u(1)=a=\lambda \cdot 1$ for some $\lambda \in \mathbf{C}^{*}$. Hence the subspace $\mathbf{C} \cdot 1$ is $G_{X}$-invariant and $G_{X}$ is reducible. It is easy to check that $G_{X} \supset Q T$.

By Theorem 2, the identity component, $G_{X}^{0}$, of $G_{X}$ has the form $G_{X}^{0}=H Q T$ where $H$ is one of the groups listed in (6).

If $n=2$ we claim that $H=\mathrm{SL}(3, \mathbf{R})$. Let $u \in \mathrm{SL}(3, \mathbf{R})$, which means that $u(1)=1, u$ preserves the space of hermitian matrices of trace 0 , and $\operatorname{det}(u)=1$. Each $x \in X$ can be written uniquely as $x=\lambda \cdot 1+x_{0}$ with $x_{0} \in X$ and $\operatorname{tr}\left(x_{0}\right)=0$. Furthermore, $x_{0}=a+i b$ where $a$ and $b$ are commuting hermitian matrices. As $a$ and $b$ have real traces and $\operatorname{tr}\left(x_{0}\right)=0$, we must have $\operatorname{tr}(a)=\operatorname{tr}(b)=0$. Since $a$ and $b$ are unitarily diagonalizable, it is easy to see that $a b=b a$ implies that $a$ and $b$ are linearly dependent over $\mathbf{R}$. Since $u\left(x_{0}\right)=u(a)+i u(b)$, it follows that $u\left(x_{0}\right)$, and also $u(x)$, is a normal matrix. As $\operatorname{SL}(3, \mathbf{C}) \not \subset G_{X}$, our claim follows by inspecting the list (6).

From now on let $n>2$. We claim that $G_{X} \not \supset \mathrm{SO}\left(n^{2}-1\right)$. Indeed let $x$ and $y$ be the diagonal matrices

$$
x=\operatorname{diag}(1,-1,0, \ldots, 0), \quad y=\operatorname{diag}(1,1,-2,0, \ldots, 0),
$$

and let

$$
z=\left(\begin{array}{cc}
0 & \sqrt{3} \\
\sqrt{3} & 0
\end{array}\right) \oplus 0_{n-2}
$$

These are hermitian matrices of trace 0 . As $\operatorname{tr}(x y)=\operatorname{tr}(x z)=0$ and $\operatorname{tr}\left(y^{2}\right)=$ $\operatorname{tr}\left(z^{2}\right)=6$, there exists $u \in \mathrm{SO}\left(n^{2}-1\right)$ such that $u(x)=x$ and $u(y)=z$. As $x y=y x, x+i y$ is normal. On the other hand $x z \neq z x$, and so $u(x+i y)=x+i z$ is not normal. Hence $u \notin G_{X}$, and our claim is proved.

It is clear that $G_{X} \not \supset G$. By inspecting the list (6), we infer that $H=G_{0}$ if $n \neq 4$. We claim that this is also true for $n=4$. It suffices to show that $\Lambda_{0}$ and $\Lambda_{1}$ are not contained in $G_{X}$. Let $\left\{v_{i}\right\}$ be the basis of $K_{4}$ introduced in Section 2 . Since $\operatorname{tr}\left(v_{1}^{*} v_{3}\right)=\operatorname{tr}\left(v_{1}^{*} v_{6}\right)=0$ and $\operatorname{tr}\left(v_{3}^{*} v_{3}\right)=\operatorname{tr}\left(v_{6}^{*} v_{6}\right)=2$, there exists $a \in \mathrm{SU}(6)$ such that $a\left(v_{1}\right)=v_{1}$ and $a\left(v_{6}\right)=v_{3}$. As $v_{1} f\left(v_{6}\right)-v_{6} f\left(v_{1}\right)=v_{1}^{2}-v_{6}^{2}$ is normal, while

$$
\rho(a)\left(v_{1} f\left(v_{6}\right)-v_{6} f\left(v_{1}\right)\right)=v_{1} f\left(v_{3}\right)-v_{3} f\left(v_{1}\right)=-v_{1} v_{4}-v_{3} v_{6}
$$

is not, we infer that $\rho(a) \notin G_{X}$, and so $G_{X} \not \supset \Lambda_{0}$.

The matrices

$$
\begin{array}{lll}
w_{1}=v_{1}+v_{6}, & w_{2}=v_{2}-v_{5}, & w_{3}=v_{3}+v_{4}, \\
w_{4}=i\left(v_{1}-v_{6}\right), & w_{5}=i\left(v_{2}+v_{5}\right), & w_{6}=i\left(v_{3}-v_{4}\right),
\end{array}
$$


form a basis of the real subspace of $K_{4}$ defined by the equation $f(x)=\bar{x}$. If $x$ and $y$ belong to this subspace, then it is easy to check that $x f(y)-y f(x)$ is a skew-hermitian matrix. Let $a \in \mathrm{SL}(6, \mathbf{R})$ be such that

$$
a\left(w_{1}\right)=w_{1}, \quad a\left(w_{2}\right)=w_{1}+w_{2}, \quad a\left(w_{4}\right)=w_{4}, \quad a\left(w_{5}\right)=w_{6} .
$$

Since

$$
\begin{aligned}
w_{1} f\left(w_{4}\right)-w_{4} f\left(w_{1}\right) & =-w_{1} w_{4}-w_{4} w_{1}=-2 w_{1} w_{4} \\
& =2 i \cdot \operatorname{diag}(1,1,-1,-1), \\
w_{2} f\left(w_{5}\right)-w_{5} f\left(w_{2}\right) & =-w_{2} w_{5}-w_{5} w_{2}=-2 w_{2} w_{5} \\
& =2 i \cdot \operatorname{diag}(1,-1,1,-1),
\end{aligned}
$$

the matrix

$$
x=\left[w_{1} f\left(w_{4}\right)-w_{4} f\left(w_{1}\right)\right]+i\left[w_{2} f\left(w_{5}\right)-w_{5} f\left(w_{2}\right)\right]
$$

is normal. On the other hand, it is easy to check that the $(1,3)$ entry of the matrix

$$
\left(w_{1}+w_{2}\right) f\left(w_{6}\right)-w_{6} f\left(w_{1}+w_{2}\right)=-\left(w_{1}+w_{2}\right) w_{6}-w_{6}\left(w_{1}+w_{2}\right)
$$

is not 0 . Consequently this matrix does not commute with $w_{1} f\left(w_{4}\right)-w_{4} f\left(w_{1}\right)$, and so the matrix

$$
\rho(a)(x)=\left[w_{1} f\left(w_{4}\right)-w_{4} f\left(w_{1}\right)\right]+i\left[\left(w_{1}+w_{2}\right) f\left(w_{6}\right)-w_{6} f\left(w_{1}+w_{2}\right)\right]
$$

is not normal. Thus $\rho(a) \notin G_{X}$, and so $G_{X} \not \supset \Lambda_{1}$.

Since $\tau \in G_{X}$, the assertion of the theorem follows from Theorem 4 .

\section{REFERENCES}

1. D. Ž. Đoković and V. P. Platonov, Algebraic groups and linear preserver problems, C. R. Acad. Sci. Paris Sér. I Math. 317 (1993), 925-930. MR 94i:20080

2. M. Goto and F. Grosshans, Semisimple Lie algebras, Dekker, New York, 1978. MR 58:28084

3. M. Goto, On an arcwise connected subgroup of a Lie group, Proc. Amer. Math. Soc. 20 (1969), 157-162. MR 38:2244

4. S. Helgason, Differential geometry, Lie groups, and symmetric spaces, Academic Press, New York, 1978. MR 80k:53081

5. V. P. Platonov and D. Ž. Đoković, Linear preserver problems and algebraic groups, Preprint 94-029, Discrete Structures in Math., University of Bielefeld, 1994.

6. A survey of linear preserver problems, Linear and Multilinear Algebra 33 (1992), 1-130.

Department of Pure Mathematics, University of Waterloo, Waterloo, Ontario, N2L 3G1 CANADA

E-mail address: dragomir@herod.uwaterloo.ca 